\title{
Dispersion relations of Yukawa fluids at weak and moderate coupling
}

\author{
Sergey Khrapak $\oplus^{1,2,3}$ and Lénaïc Couëdel ${ }^{4,5}$ \\ ${ }^{1}$ Institut für Materialphysik im Weltraum, Deutsches Zentrum für Luft- und Raumfahrt (DLR), 82234 Weßling, Germany \\ ${ }^{2}$ Joint Institute for High Temperatures, Russian Academy of Sciences, 125412 Moscow, Russia \\ ${ }^{3}$ Bauman Moscow State Technical University, 105005 Moscow, Russia \\ ${ }^{4}$ Physics and Engineering Physics Department, University of Saskatchewan, 116 Science Place, S7N 5E2 Saskatoon, Saskatchewan, Canada \\ ${ }^{5}$ CNRS, Aix-Marseille Université, Laboratoire PIIM, UMR 7345, 13397 Marseille cedex 20, France
}

(Received 26 April 2020; revised 18 August 2020; accepted 19 August 2020; published 21 September 2020)

\begin{abstract}
In this paper we compare different theoretical approaches to describe the dispersion of collective modes in Yukawa fluids when the interparticle coupling is relatively weak, so that the kinetic and potential contributions to the dispersion relation compete with each other. A thorough comparison with the results from molecular dynamics simulation allows us to conclude that, in the investigated regime, the best description is provided by the sum of the generalized excess bulk modulus and the Bohm-Gross kinetic term.
\end{abstract}

DOI: 10.1103/PhysRevE.102.033207

\section{INTRODUCTION}

There has been substantial evidence that the quasilocalized charge approximation (QLCA), also known as the quasicrystalline approximation (QCA), describes rather well the long-wavelength portion of the dispersion relations of collective excitations in strongly coupled Yukawa fluids [1-6], including the one-component plasma (OCP) limit [7-10]. The purpose of this study is to investigate the dispersion relation of Yukawa fluids at moderate coupling.

This regime corresponds to the "true" fluid situation, where no small parameter is present. At weak coupling, interactions between particles provide a small correction to the conventional multicomponent plasma dispersion relation. At strong coupling, QLCA (QCA) does a rather good job, and the kinetic corrections are numerically small and can normally be neglected. What can an appropriate theoretical approximation be between these two limits?

We provide an answer to this question below. Since well-defined transverse (shear) modes in fluids are normally supported only in the vicinity of the fluid-solid phase transition (strong coupling regime) $[2,11-14]$ we concentrate on the longitudinal mode here. The transverse mode will be mentioned only briefly, to the extent necessary for the understanding of the proposed approximations.

Recently, the evolution of the longitudinal sound velocity of Yukawa systems from the weak to the strong-coupling regimes has been studied in detail in Ref. [15]. Sound velocity can be related to thermodynamic quantities, and hence the knowledge of an appropriate equation of state can solve the problem. Here we analyze the entire dispersion curves, not only their long-wavelength asymptotes.

Extensive molecular dynamics (MD) simulations were performed to obtain the dispersion relations of weakly and moderately coupled Yukawa fluids. Theoretical approximations applicable to this regime are discussed, and the most suitable is identified. It turns out that the generalized excess bulk modulus supplemented by the Bohm-Gross kinetic term provides a particularly good theoretical description of the numerically obtained dispersion curves.

\section{YUKAWA FLUIDS}

Historically, interest in classical systems of particles interacting via the repulsive Yukawa (screened Coulomb or Debye-Hückel) potential was mainly related to modeling charges immersed in a polarizable background, e.g., electron-ion plasma and charge-stabilized colloidal dispersions [16-18]. More recently, the Yukawa potential has been extensively used as a first approximation to model interactions between macroscopic particles in complex (dusty) plasmas [19-24]. In a more general context, the Yukawa potential represents an important example of soft repulsive interactions operating in various soft matter systems.

In Yukawa systems particles are interacting via the pairwise potential of the form

$$
\phi(r)=\left(Q^{2} / r\right) \exp (-r / \lambda),
$$

where $Q$ is the particle charge and $\lambda$ is the screening length. Such a system is fully characterized by the two dimensionless parameters: the coupling parameter $\Gamma=Q^{2} / a T$ and the screening parameter $\kappa=a / \lambda$, where $a=(4 \pi n / 3)^{-1 / 3}$ is the Wigner-Seitz radius, $T$ is the temperature in energy units $\left(k_{\mathrm{B}}=1\right)$, and $n$ is the density. Conventionally, the system is referred to as strongly coupled (nonideal) when $\Gamma \gg 1$, that is, when the Coulomb interaction energy exceeds considerably the kinetic energy [more precisely, when $\phi(a) \gg T$, so that screening is accounted for]. The opposite limit $\Gamma \ll 1$ corresponds to the weakly coupled (ideal) regime.

The phase diagram of three-dimensional Yukawa systems in $(\kappa, \Gamma)$ plane is shown in Fig. 1. The solid curve smoothly connect the melting points data obtained from the free energy consideration and tabulated in Ref. [25] (accurate analytical 


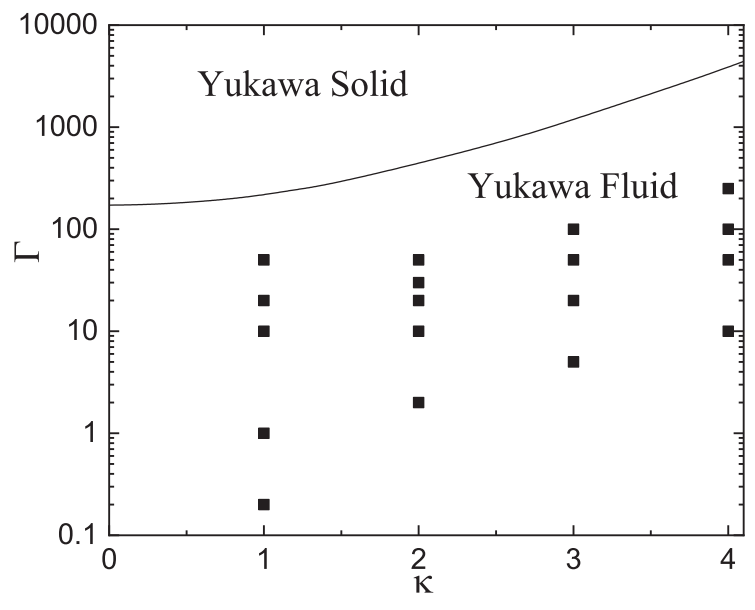

FIG. 1. Phase diagram of Yukawa systems in a $(\kappa, \Gamma)$ plane. The solid curve corresponds to the fluid-solid (melting) phase transition according to the data from Ref. [25]. Symbols correspond to the phase state points investigated in this work.

fits are also available [26,27]). We consider a moderate screening regime with $1 \leqslant \kappa \leqslant 4$, which is particularly relevant for complex plasma experiments in gas discharges. In the considered range of $\kappa$, a Yukawa fluid first freezes into the bcc lattice. The fcc lattice can be also stable, but either at higher $\kappa$ or at higher $\Gamma$ (only for $\kappa \gtrsim 1$ ) [25]. It should be noted that in real experiments with complex plasmas, a metastable hcp lattice can be present or even be a dominant constituent of the solid phase [28-31]. Symbols in the theoretical phase diagram depicted in Fig. 1 correspond to state points investigated in this work.

\section{THEORETICAL APPROACHES AND STRATEGY}

\section{A. Fluid and kinetic descriptions}

A simple fluid description leads, in the weakly coupled regime, to the dispersion relation of the form [21]

$$
\omega^{2}=\frac{\omega_{\mathrm{p}}^{2} k^{2} \lambda^{2}}{1+k^{2} \lambda^{2}}+\gamma k^{2} v_{\mathrm{T}}^{2}
$$

where $\omega$ is the frequency, $k$ is the wave vector, $\omega_{\mathrm{p}}=$ $\sqrt{4 \pi Q^{2} n / m}$ is the plasma frequency, $v_{\mathrm{T}}=\sqrt{T / m}$ is the thermal velocity, and $\gamma$ is the effective polytrope index. If the last (kinetic) term is neglected, then the resulting dispersion relation is usually referred to as the dust acoustic wave (DAW) dispersion relation [20,32-34]. At long wavelengths the acoustic dispersion is recovered, $\omega \simeq k c_{\mathrm{DA}}$ with $c_{\mathrm{DA}}=$ $\omega_{\mathrm{p}} \lambda$.

The kinetic description allows us to fix the numerical coefficient in the kinetic term. The result is that at weak coupling and long wavelengths we have to chose $\gamma=3$. The term $3 k^{2} v_{\mathrm{T}}^{2}$ is often referred to as the Bohm-Gross term, after Ref. [35], and we follow this tradition here. Although the kinetic description is applicable only in the ideal plasma limit, when correlations between particles are absent (weak coupling), we will see below that the magnitude of the kinetic term itself is not changed much even at moderate (and possibly at strong) coupling.
Expressed in reduced units the fluid (kinetic) dispersion relation becomes

$$
\frac{\omega^{2}}{\omega_{\mathrm{p}}^{2}}=\frac{q^{2}}{q^{2}+\kappa^{2}}+\gamma \frac{q^{2}}{3 \Gamma},
$$

where $q=k a$. Further details concerning the fluid and kinetic description of weakly coupled Yukawa fluids can be found in the Supplemental Material [36], which includes Refs. [37-40].

\section{B. Frequency moments and QLCA}

The second frequency moments of the longitudinal and transverse current correlation functions are defined as [41]

$$
\omega_{L}^{2}(k)=3 k^{2} v_{\mathrm{T}}^{2}+\frac{n}{m} \int \frac{\partial^{2} \phi(r)}{\partial z^{2}} g(r)[1-\cos (\mathbf{k z})] d \mathbf{r}
$$

and

$$
\omega_{T}^{2}(k)=k^{2} v_{\mathrm{T}}^{2}+\frac{n}{m} \int \frac{\partial^{2} \phi(r)}{\partial x^{2}} g(r)[1-\cos (\mathbf{k z})] d \mathbf{r} .
$$

The subscripts $L$ and $T$ refer to the longitudinal and transverse modes, respectively. The expressions above contain the kinetic (first term) and potential (or excess) contributions (second term). The potential contribution is expressed in terms of the pairwise interparticle interaction potential $\phi(r)$ and equilibrium radial distribution function (RDF) $g(r)$. The kinetic contribution to the longitudinal mode is formally given by the same Bohm-Gross term.

The QLCA and QCA approximations [5,8,42,43] tell us that the dispersion relations of the longitudinal and transverse modes at strong coupling are given by the potential contributions in Eqs. (4) and (5). An exceptionally enlightening physical derivation demonstrating why it should be approximately so is due to Hubbard and Beeby [42]. Thus, QLCA approach does not take into account direct thermal effects. This is not a problem at strong coupling, because kinetic terms are numerically small in this regime. At weaker coupling kinetic effects should be accounted for to improve the accuracy of the QLCA $[13,44]$.

The explicit expressions for $\omega_{L}(k)$ and $\omega_{T}(k)$ for the Yukawa interaction potential can be found elsewhere [3-5]. In the complete absence of correlations, for $g(r)=1$, the conventional DAW dispersion relation is recovered for the longitudinal mode

$$
\omega_{L}^{2}=\frac{\omega_{\mathrm{p}}^{2} q^{2}}{q^{2}+\kappa^{2}}+3 k^{2} v_{\mathrm{T}}^{2}
$$

which coincides with Eq. (3) with $\gamma=3$. Note that the kinetic term vanishes at $T \rightarrow 0$, and we recover the conventional (cold plasma limit) DAW dispersion relation. This situation is, however, not internally consistent with the assumption of no correlations, $g(r)=1$.

In the absence of correlations, the potential contribution to the transverse mode is identically zero, so that

$$
\omega_{T}^{2}=k^{2} v_{\mathrm{T}}^{2}
$$

Even though it follows from Eq. (7) that the transverse frequency is nonzero due to the presence of the kinetic term, this 
mode is not supported in weakly coupled gases and moderately coupled fluids. It will not be considered further. Recent theoretical results regarding the onset and simple description of transverse waves in strongly coupled Yukawa fluids can be found elsewhere $[6,14]$. The process of shear rigidity emergence with increasing coupling and interparticle correlations in Yukawa systems, starting from the weakly coupled gaseous regime, has been also investigated [45].

\section{Generalized bulk modulus}

The dispersion relations resulting from the frequency moments and QLCA approaches can be also expressed in terms of generalized high-frequency (instantaneous) bulk $\left(K_{\infty}\right)$ and shear $\left(G_{\infty}\right)$ moduli as follows [46]:

$$
\omega_{T}^{2}(k)=\frac{k^{2}}{m n} G_{\infty}(k)
$$

and

$$
\omega_{L}^{2}(k)=\frac{k^{2}}{m n}\left[K_{\infty}(k)+\frac{4}{3} G_{\infty}(k)\right] .
$$

In the long-wavelength limit $(k \rightarrow 0), K_{\infty}$ and $G_{\infty}$ become just conventional instantaneous fluid elastic moduli [47,48]. The sum $K_{\infty}+\frac{4}{3} G_{\infty}=M_{\infty}$ is known as the longitudinal modulus. The essential physics behind the relevance of infinite frequency elastic moduli in the fluid regime is the following. If a perturbation is suddenly applied to a dense strongly coupled fluid (not too far from the fluid-solid phase transition), its initial response would not be very much different from that of a solid. It will respond elastically with the longitudinal response that depends on both the bulk and shear elastic moduli. This emphasizes the solidlike properties of strongly coupled fluids.

As the interparticle coupling weakens and the role of interparticle correlations diminishes, the transverse mode becomes irrelevant. It is tempting to assume that the longitudinal mode dispersion would decouple from the transverse one and depend on the generalized instantaneous bulk modulus alone, that is, $\omega^{2}(k) \simeq \omega_{L}^{2}(k)-\frac{4}{3} \omega_{T}^{2}(k)$. In fact, a similar conjecture has been demonstrated to result in a meaningful approximation for weakly and moderately coupled classical Coulomb fluids in two dimensions [10]. For a three-dimensional Coulomb fluid (one-component plasma), the dispersion relation of the form

$$
\omega^{2}=3 k^{2} v_{\mathrm{T}}^{2}+\frac{k^{2}}{m n} \Delta K_{\infty}(k)
$$

has been demonstrated to capture correctly the onset of negative dispersion (the point where $d \omega / d k$ starts to be negative at $k \rightarrow 0$ ) [49]. Here $\Delta K_{\infty}$ is the excess component of the generalized bulk modulus. Motivated by the relative success of this approximation we have chosen to compare it with the results of MD simulations.

\section{Strategy}

We have performed extensive MD simulations to determine the dispersion relation of the longitudinal collective mode for a broad parameter regime (see Fig. 1). Direct comparison with the predictions of approximations described in this section is used to test their relative success. In particular, we take three approximations: (i) weakly coupled expression from the fluid, kinetic, and frequency moments approaches, Eq. (3); (ii) second frequency moment of the longitudinal current correlation function (4) keeping the Bohm-Gross kinetic term; and (iii) expression (10) based on the generalized instantaneous bulk modulus complemented with the Bohm-Gross kinetic term. The best choice among the considered approximations will be then identified.

\section{NUMERICAL SIMULATIONS}

The simulations were performed on graphics processing unit (NVIDIA Quadro P2000) using the HOOMD-blue software [50,51]. We used $N=55296$ Yukawa particles in a cubic box with the periodic boundary conditions. The cutoff radius for the potential has been chosen as $L_{\text {cut }}=9 \lambda_{D}$. The numerical time step was set to $\Delta t \simeq 2 \times 10^{-3} \omega_{p}^{-1}$. The simulations were performed in the canonical $N V T$ ensemble with the Langevin thermostat at a temperature corresponding to the desired target coupling parameter $\Gamma$.

The system was first equilibrated for $1.25 \times 10^{6}$ time steps with a drag coefficient $\gamma \simeq 2 \times 10^{-2} \omega_{p}$. The drag coefficient was then reduced to $\gamma \simeq 2 \times 10^{-4} \omega_{p}$, and the system was run for another 350000 time steps. Finally the particle positions and trajectories were saved every 100 time steps for 180000 time steps (except for $\kappa=4$, for which the trajectories were saved every 400 time steps for 720000 time steps in order to resolve more accurately the low-frequency fluctuations of such a system).

The particle current was then calculated:

$$
\mathbf{J}(\mathbf{k}, t)=\sum_{j=1}^{N} \mathbf{v}_{j}(t) \exp \left[\imath \mathbf{k} \cdot \mathbf{r}_{j}(t)\right],
$$

where $\mathbf{v}_{j}(t)$ and $\mathbf{r}_{j}(t)$ are the velocity and position of the $j$ th particle, respectively, and $\mathbf{k}$ is the wave vector. The Fourier transform in time was performed to obtain the current fluctuation spectra. The particle positions were also used every 400 time steps to extract the accurate radial distribution functions $g(r)$.

To obtain the dispersion relation $\omega_{l}(k)$, the longitudinal current fluctuation spectrum $C_{l}(k, \omega)$ was fitted to the doubleLorentzian form [10,52]:

$$
C_{l}(k, \omega) \propto \frac{\gamma_{l}(k)}{\left[\omega-\omega_{l}(k)\right]^{2}+\gamma_{l}(k)^{2}}+\frac{\gamma_{l}(k)}{\left[\omega+\omega_{l}(k)\right]^{2}+\gamma_{l}(k)^{2}},
$$

where $\gamma_{l}$ denotes the damping rate of the longitudinal mode.

\section{RESULTS}

We start by analyzing the two very weakly coupled state points characterized by $\kappa=1$ and the two $\Gamma$ values, $\Gamma=0.2$ and $\Gamma=1.0$. For these state points the contribution from particle-particle interactions is very small, and this gives us the opportunity to concentrate on the behavior of the kinetic contribution to the dispersion relation.

The ratio $\omega / \omega_{\mathrm{p}} q$ versus $q$, in a very extended range of $q$, is plotted in Fig. 2. From comparison with Eq. (3) we observe that the effective coefficient $\gamma$ is between 2 and 3 . 


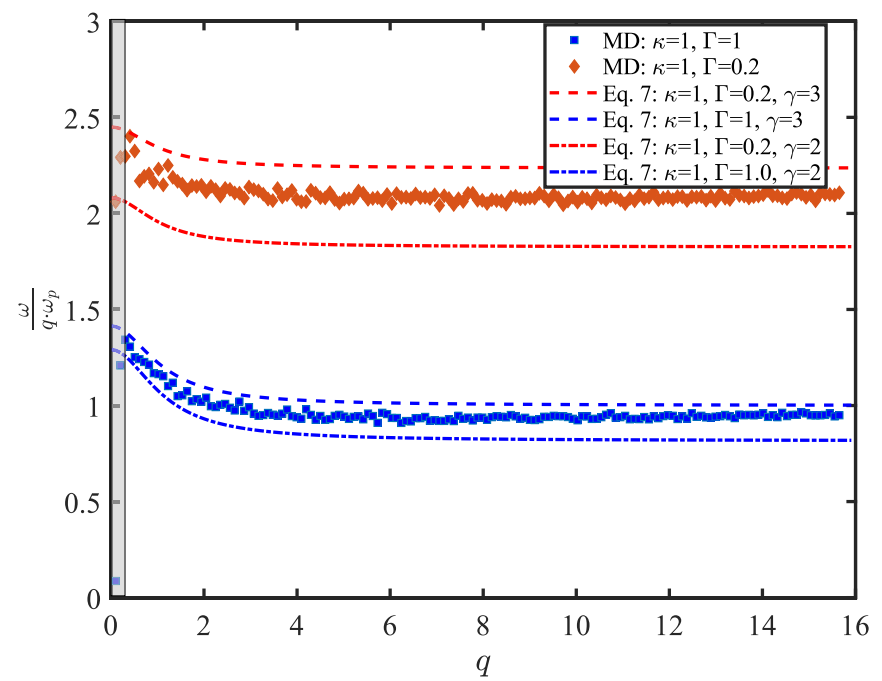

FIG. 2. Ratio $\omega / q \omega_{\mathrm{p}}$ versus the reduced wave number $q=k a$ for the two weakly coupled state points $(\kappa=1, \Gamma=0.2)$ and $(\kappa=$ $1, \Gamma=1)$. The shaded area corresponds to the regime of insufficient statistics due to finite simulation volume [this approximately corresponds to $q \lesssim 2 \pi /(L / 2) \simeq 0.2$, where $L$ is the size of the simulation box]. The dashed (dotted) curves correspond to Eq. (3) with $\gamma=3$ $(\gamma=2)$.

Actually, it is somewhat closer to 3, as the kinetic theory and frequency moments expressions predict. Moreover, it remains constant to a very good accuracy in the considered very wide range of $q$. The region of very low $q$ is not well resolved, but here the reduced sound velocity seems to approach smoothly $\sqrt{6} \simeq 2.45$ for $\Gamma=0.2$ and $\sqrt{2} \simeq 1.41$ for $\Gamma=1$, as Eq. (3) with $\gamma=3$ predicts. Thus, although we are not able to provide the exact value of the coefficient $\gamma$, we observe that the Bohm-Gross term $3 k^{2} v_{\mathrm{T}}^{2}$ delivers a reasonable approximation in the range of $q$ investigated. The individual particle limit with $\omega^{2} \simeq 2 k^{2} v_{\mathrm{T}}^{2}[41,52]$ is not reached in our simulations; see also the Appendix.

The dispersion relations obtained in our numerical experiment are shown in Figs. 3-6. Here the symbols correspond to $\omega_{l}(q)$ and the vertical lines ("error bars") mark $\omega_{l}(q) \pm \gamma_{l}(q)$, as obtained from the fits using Eq. (12). The data points corresponding to the very long wavelength range (gray area in Fig. 2) are not very reliable because they are influenced by the finite size effects. We do not add the gray area in Figs. 3-6 to not overload the plots. Theoretical curves are also plotted for the purpose of comparison. The following main trends can be summarized.

In the weakly coupled regime all the theoretical approximations agree very well with the numerical data. Here the interparticle correlations are small, and excess terms from the weakly coupled fluid and strongly coupled QCA approaches are nearly identical. There is also no difference between the generalized longitudinal and bulk moduli, because the excess shear modulus vanishes in the weakly coupled limit.

In the moderately coupled regime, the excess component of the generalized bulk modulus complemented by the Bohm-Gross kinetic term provides the best agreement with numerical data among the approximations considered. The
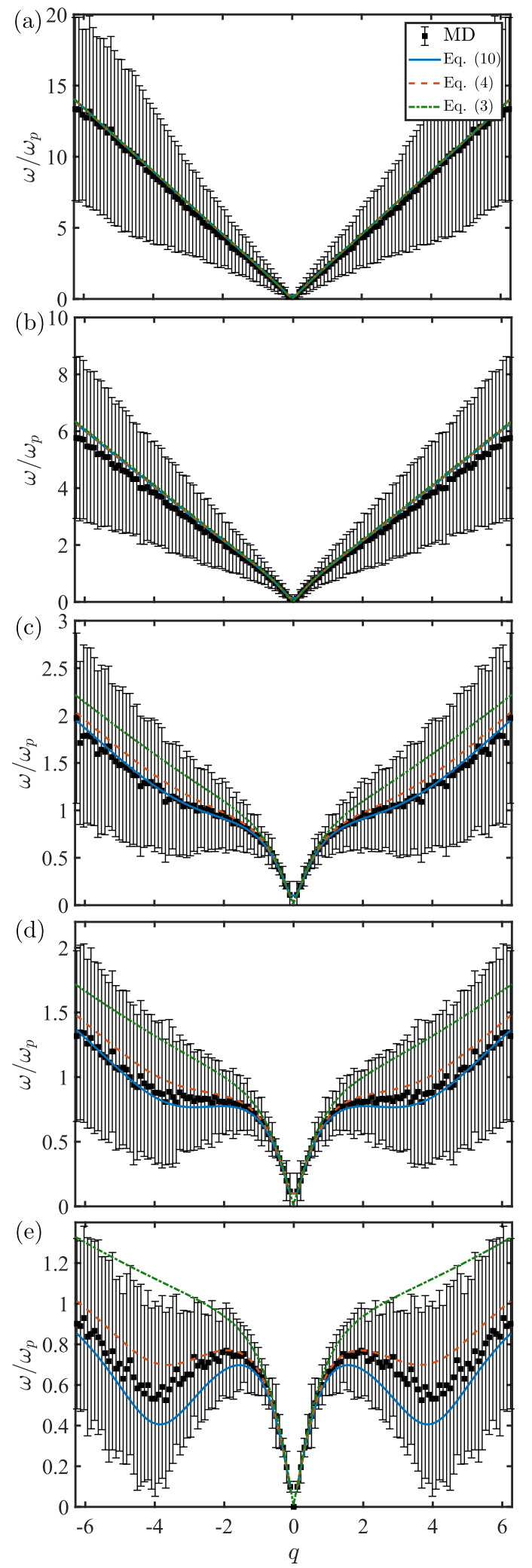

FIG. 3. Dispersion relations in Yukawa fluids with $\kappa=1$. Symbols correspond to numerical results. Curves denote theoretical approximations compared in this work (see the legend). (a) $\Gamma=0.2$, (b) $\Gamma=1.0$, (c) $\Gamma=10$, (d) $\Gamma=20$, (e) $\Gamma=50$.

second frequency moment expression (4) somewhat overestimates the frequency. 

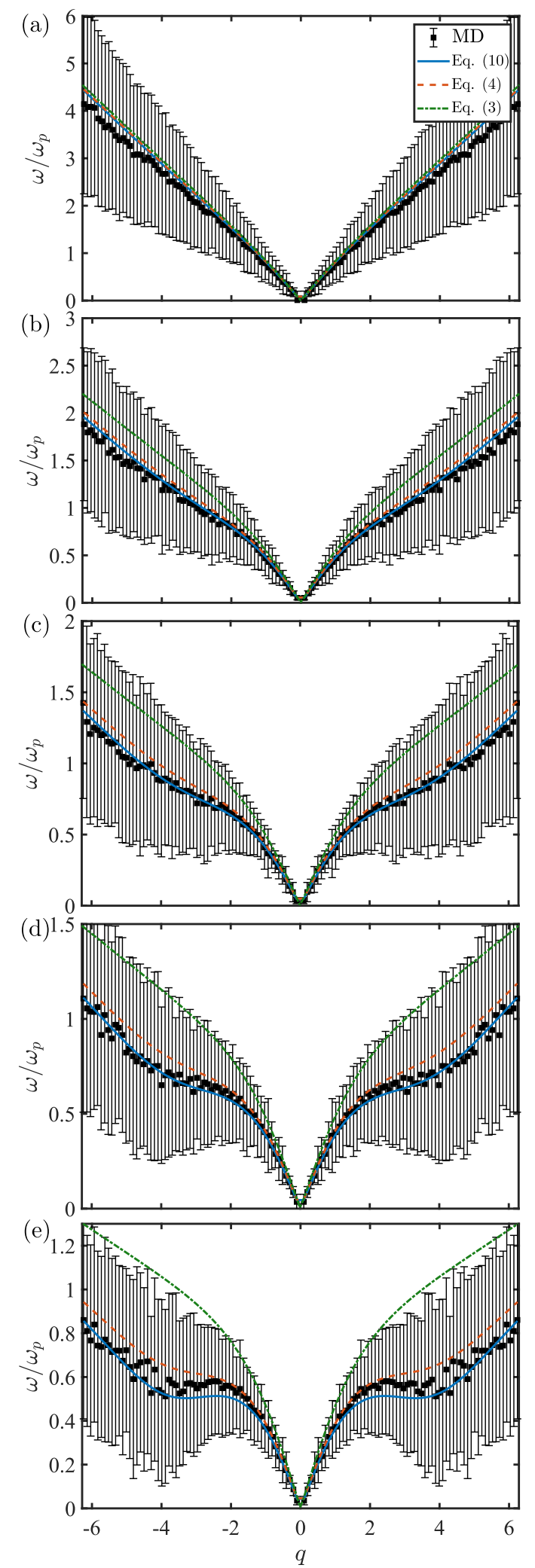

FIG. 4. Dispersion relations in Yukawa fluids with $\kappa=2$. Notation is the same as in Fig. 3. (a) $\Gamma=2.0$, (b) $\Gamma=10$, (c) $\Gamma=20$, (d) $\Gamma=30$, (e) $\Gamma=50$.

When approaching the strongly coupled regime, none of the approximations considered allow us to describe numerical data accurately in the entire $q$ range investigated. The
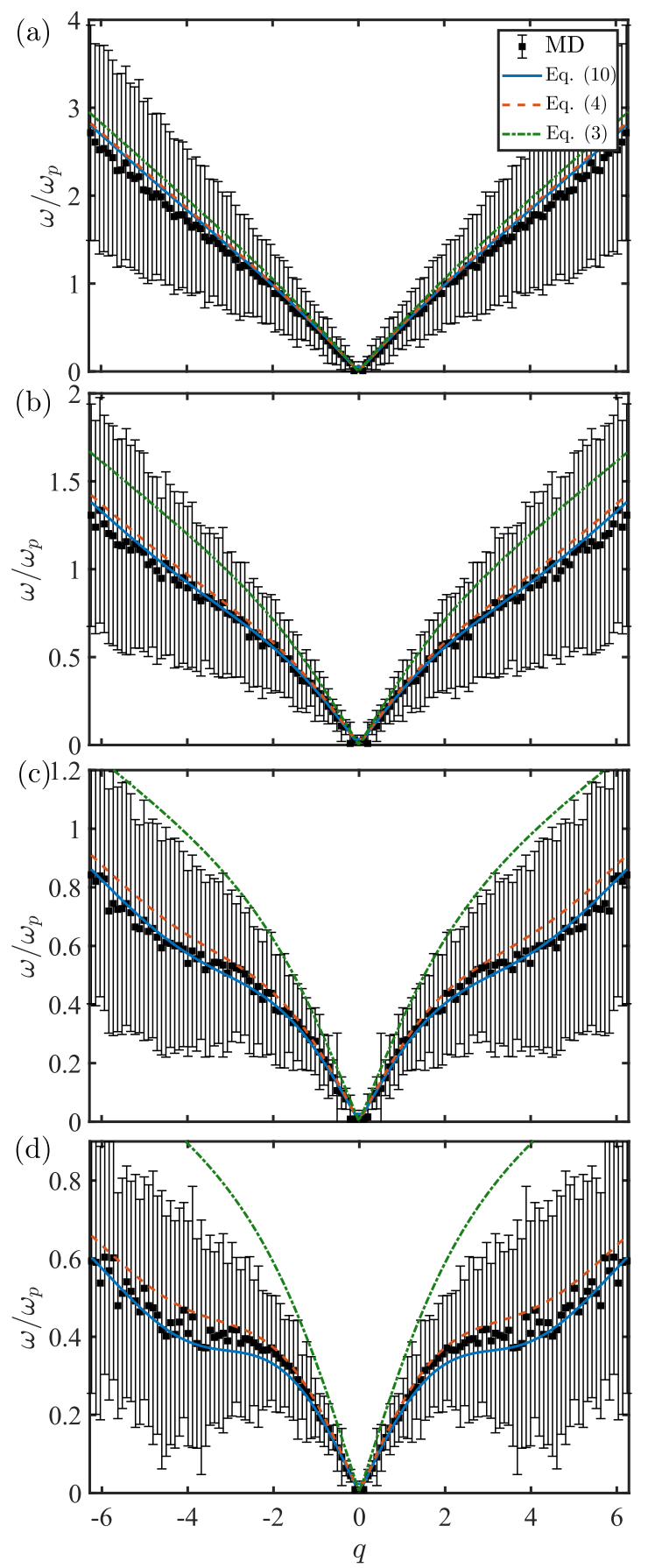

FIG. 5. Dispersion relations in Yukawa fluids with $\kappa=3$. Notation is the same as in Fig. 3. (a) $\Gamma=5.0$, (b) $\Gamma=20$, (c) $\Gamma=50$, (d) $\Gamma=100$.

second frequency moment (4) expression deviates to higher frequencies as $q$ increases. The generalized bulk modulus expression underestimate the frequency near the first minimum. This becomes particularly clear for the state point with $\kappa=1$ and $\Gamma=50$. Similar tendency has been observed in classical two-dimensional Coulomb fluids [10]. In the longwavelength regime, $q \lesssim 3$, the second frequency moment, and hence QLCA and QCA, provide adequate description at strong coupling, as has been already noted in the Introduction. At even longer wavelengths $(q \ll 1)$ the difference between 

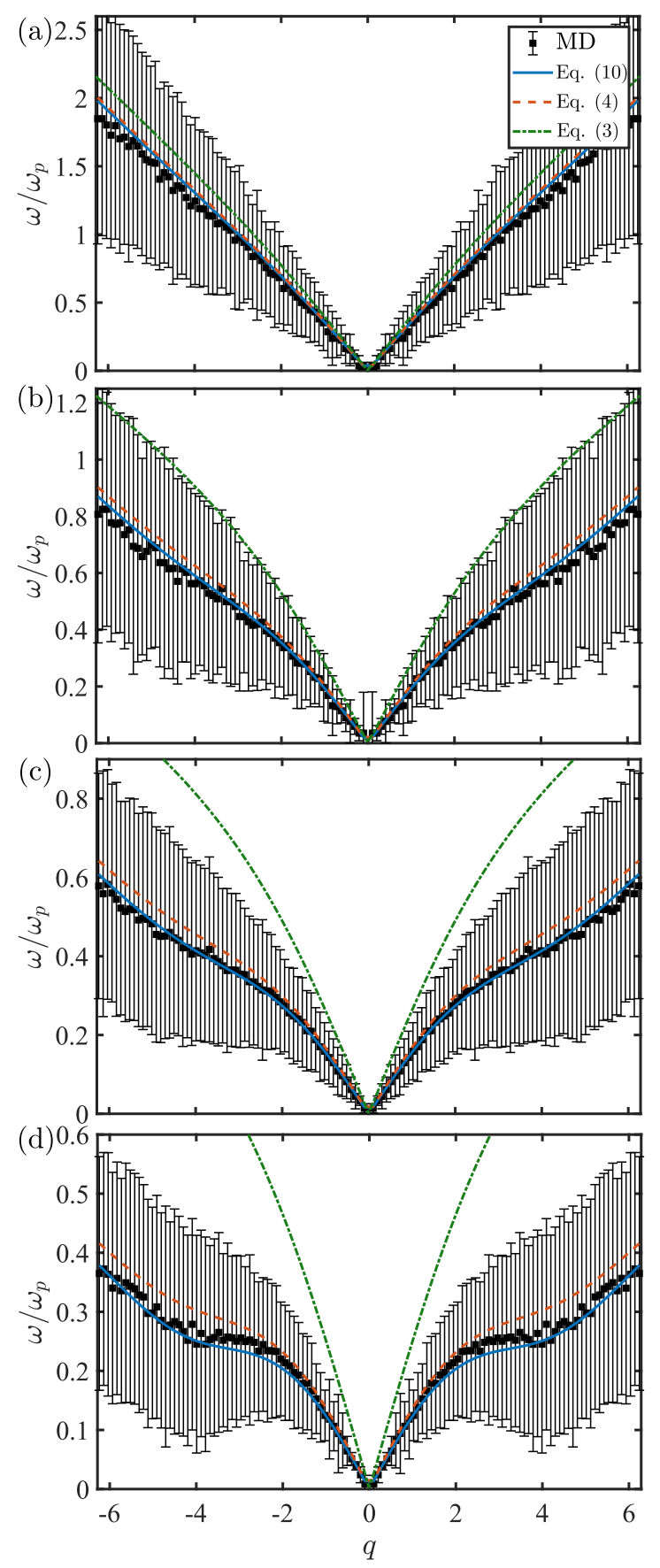

FIG. 6. Dispersion relations in Yukawa fluids with $\kappa=4$. Notation is the same as in Fig. 3. (a) $\Gamma=10$, (b) $\Gamma=50$, (c) $\Gamma=100$, (d) $\Gamma=250$.

the second frequency moment and generalized bulk modulus expressions practically disappears, because of the inequality $G_{\infty} \ll M_{\infty} \simeq K_{\infty}$ and acoustic character of the dispersion.

The appearance of the minimum in the dispersion relation can serve as a pragmatic demarcation between the moderately and strongly coupled regimes. This can have relations with the crossover between gaslike and fluidlike behavior, the concept known as the "Frenkel line," on the phase diagram [53].

Regarding the weakly coupled fluid and kinetic approximations, they become particularly inappropriate when the screening parameter increases. This could be expected. Strong coupling effects are known to affect only weakly the magnitude of the sound velocity at $\kappa \lesssim 1$ but lead to its considerable decrease for higher $\kappa$ [3]; see in particular Fig. 4 in Ref. [54] and Fig. 5 in Ref. [55].

\section{CONCLUSION}

In this paper we have addressed the question regarding what determines the dispersion relations of Yukawa fluids at moderate coupling, when kinetic and potential contributions to the dispersion relations are of similar magnitude. Three theoretical approaches have been compared with the results from extensive MD simulations. Among these, an empirical expression combining the generalized excess bulk modulus with the Bohm-Gross kinetic term provides the best agreement with numerical results at weak and moderate coupling. The approach to the strong coupling regime is signaled by the appearance of a pronounced minimum in the dispersion relation. In this regime none of the approximations considered allow us to describe numerical data accurately in the entire range of wave vectors.

\section{ACKNOWLEDGMENTS}

We thank H. Thomas for a careful reading of the manuscript and S. Yurchenko for useful discussions. L. Couëdel acknowledges the support of the Natural Sciences and Engineering Research Council of Canada (NSERC), RGPIN-2019-04333. Work on collective dynamics in different regimes of interparticle interaction was supported by the Russian Science Foundation, Grant No. 20-12-00356.

\section{APPENDIX: FREE-PARTICLE LIMIT}

In the limit of very large wave vectors, the current fluctuations spectra are expected to approach their free-particle

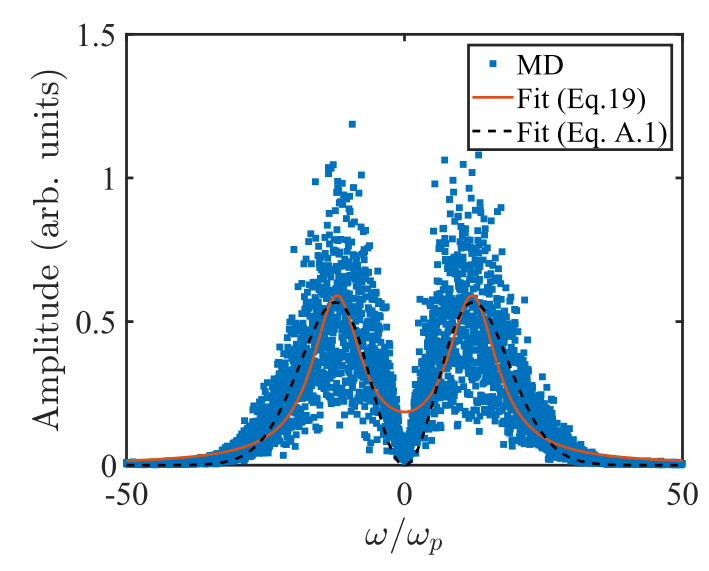

FIG. 7. Example of fitting the MD data by the double-Lorentzian and Maxwellian functions. MD data correspond to the state point with $\kappa=1$ and $\Gamma=1$, and the reduced wavelengths is $q=13$. There is some observable difference between the fits, the Maxwellian fit being more appropriate at low frequencies. However, the maximum position is about the same for both fits. 


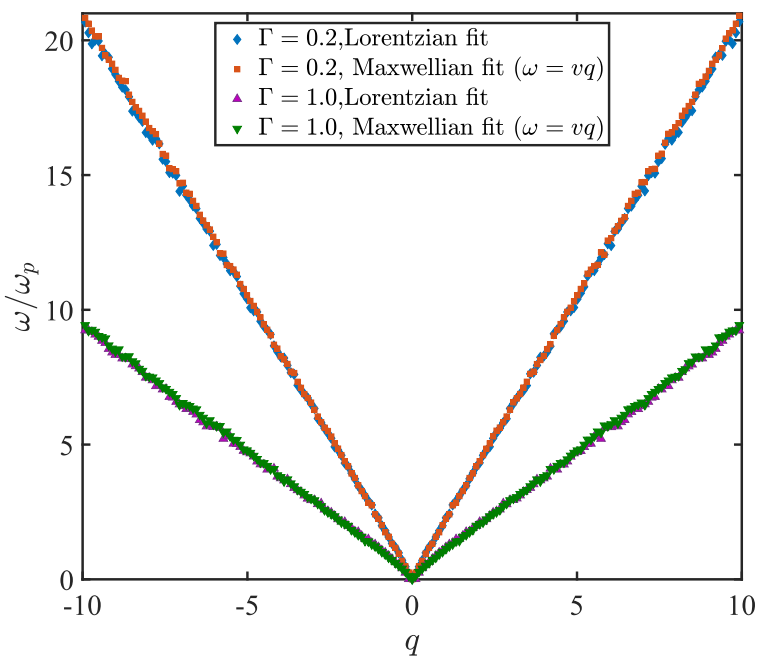

FIG. 8. Reduced frequency $\omega / \omega_{\mathrm{p}}$ versus the reduced wave number $q=k a$ for the two weakly coupled state points, $(\kappa=1, \Gamma=0.2)$ and $(\kappa=1, \Gamma=1)$. The results of analysis using double-Lorentzian and Maxwellian fits are almost indistinguishable. limiting expressions. For the longitudinal spectrum this corresponds to the Maxwellian shape [41,52]

$$
C_{l}(q, \omega) \propto\left(\frac{\omega}{q}\right)^{2} \exp \left(-\frac{m \omega^{2} a^{2}}{2 T q^{2}}\right),
$$

which is peaked at $\omega a= \pm \alpha q v_{\mathrm{T}}$ with $\alpha=\sqrt{2}$. We have repeated the analysis of the two weakly coupled state points longitudinal current spectra using the Maxwellian shape and treating $\alpha$ as a free parameter. Figure 7 shows an example of fitting the MD data by the double-Lorentzian and Maxwellian functions. At low frequencies, the Maxwellian fit is clearly more appropriate (as expected), indicating that the applicability range of the double-Lorentzian fit is limited to relatively long wavelengths. Nevertheless, both fits return almost the same position of the maximum. This is further illustrated in Fig. 8, which shows that the dispersion relations constructed with the help of the double-Lorentzian and Maxwellian fits are very close. The coefficient $\alpha$ appears somewhat closer to $\sqrt{3}$ than to $\sqrt{2}$ in the regime investigated.
[1] M. Rosenberg and G. Kalman, Phys. Rev. E 56, 7166 (1997).

[2] H. Ohta and S. Hamaguchi, Phys. Rev. Lett. 84, 6026 (2000).

[3] G. Kalman, M. Rosenberg, and H. E. DeWitt, Phys. Rev. Lett. 84, 6030 (2000).

[4] Z. Donko, G. J. Kalman, and P. Hartmann, J. Phys.: Condens. Matter 20, 413101 (2008).

[5] S. A. Khrapak, B. Klumov, L. Couëdel, and H. M. Thomas, Phys. Plasmas 23, 023702 (2016).

[6] S. Khrapak and A. Khrapak, IEEE Trans. Plasma Sci. 46, 737 (2018).

[7] G. Kalman and K. I. Golden, Phys. Rev. A 41, 5516 (1990).

[8] K. I. Golden and G. J. Kalman, Phys. Plasmas 7, 14 (2000).

[9] S. A. Khrapak, AIP Adv. 7, 125026 (2017).

[10] S. A. Khrapak, N. P. Kryuchkov, L. A. Mistryukova, A. G. Khrapak, and S. O. Yurchenko, J. Chem. Phys. 149, 134114 (2018).

[11] V. Nosenko, J. Goree, and A. Piel, Phys. Rev. Lett. 97, 115001 (2006).

[12] J. Goree, Z. Donkó, and P. Hartmann, Phys. Rev. E 85, 066401 (2012).

[13] T. Ott, D. A. Baiko, H. Kählert, and M. Bonitz, Phys. Rev. E 87, 043102 (2013).

[14] S. A. Khrapak, A. G. Khrapak, N. P. Kryuchkov, and S. O. Yurchenko, J. Chem. Phys. 150, 104503 (2019).

[15] L. G. Silvestri, G. J. Kalman, Z. Donkó, P. Hartmann, M. Rosenberg, K. I. Golden, and S. Kyrkos, Phys. Rev. E 100, 063206 (2019).

[16] J. L. Barrat, J. P. Hansen, and H. Totsuji, J. Phys. C: Solid State Phys. 21, 4511 (1988).

[17] M. O. Robbins, K. Kremer, and G. S. Grest, J. Chem. Phys. 88, 3286 (1988).

[18] A. Ivlev, H. Lowen, G. Morfill, and C. P. Royall, Complex Plasmas and Colloidal Dispersions: Particle-Resolved Studies of Classical Liquids and Solids (World Scientific, Singapore, 2012).
[19] V. N. Tsytovich, Phys. Usp. 167, 57 (1997).

[20] V. E. Fortov, A. G. Khrapak, S. A. Khrapak, V. I. Molotkov, and O. F. Petrov, Phys. Usp. 47, 447 (2004).

[21] V. E. Fortov, A. Ivlev, S. Khrapak, A. Khrapak, and G. Morfill, Phys. Rep. 421, 1 (2005).

[22] S. A. Khrapak, B. A. Klumov, and G. E. Morfill, Phys. Rev. Lett. 100, 225003 (2008).

[23] V. E. Fortov and G. E. Morfill, Complex and Dusty Plasmas: From Laboratory to Space (CRC Press, Boca Raton, FL, 2009).

[24] M. Chaudhuri, A. V. Ivlev, S. A. Khrapak, H. M. Thomas, and G. E. Morfill, Soft Matter 7, 1287 (2011).

[25] S. Hamaguchi, R. T. Farouki, and D. H. E. Dubin, Phys. Rev. E 56, 4671 (1997).

[26] O. S. Vaulina and S. A. Khrapak, J. Exp. Theor. Phys. 90, 287 (2000)

[27] O. Vaulina, S. Khrapak, and G. Morfill, Phys. Rev. E 66, 016404 (2002).

[28] A. P. Nefedov, G. E. Morfill, V. E. Fortov, H. M. Thomas, H. Rothermel, T. Hagl, A. V. Ivlev, M. Zuzic, B. A. Klumov, A. M. Lipaev et al., New J. Phys. 5, 33 (2003).

[29] B. A. Klumov, Phys. Usp. 53, 1053 (2011).

[30] S. A. Khrapak, B. A. Klumov, P. Huber, V. I. Molotkov, A. M. Lipaev, V. N. Naumkin, H. M. Thomas, A. V. Ivlev, G. E. Morfill, O. F. Petrov et al., Phys. Rev. Lett. 106, 205001 (2011).

[31] S. A. Khrapak, B. A. Klumov, P. Huber, V. I. Molotkov, A. M. Lipaev, V. N. Naumkin, A. V. Ivlev, H. M. Thomas, M. Schwabe, G. E. Morfill et al., Phys. Rev. E 85, 066407 (2012).

[32] N. Rao, P. Shukla, and M. Yu, Planet. Space Sci. 38, 543 (1990).

[33] R. L. Merlino, A. Barkan, C. Thompson, and N. D'Angelo, Phys. Plasmas 5, 1607 (1998).

[34] R. L. Merlino, J. Plasma Phys. 80, 773 (2014).

[35] D. Bohm and E. P. Gross, Phys. Rev. 75, 1851 (1949).

[36] See Supplemental Material at http://link.aps.org/supplemental/ 10.1103/PhysRevE.102.033207 for details concerning the fluid and kinetic description of weakly coupled Yukawa fluids. 
[37] S. A. Khrapak and H. M. Thomas, Phys. Rev. E 91, 023108 (2015).

[38] S. A. Khrapak, Phys. Plasmas 26, 103703 (2019).

[39] S. A. Khrapak, A. G. Khrapak, A. V. Ivlev, and H. M. Thomas, Phys. Plasmas 21, 123705 (2014).

[40] L. P. Pitaevskii and E. M. Lifshitz, Physical Kinetics (Butterworth-Heinemann, Oxford, 2012).

[41] U. Balucani and M. Zoppi, Dynamics of the Liquid State (Clarendon Press, Oxford, 1995).

[42] J. Hubbard and J. L. Beeby, J. Phys. C: Solid State Phys. 2, 556 (1969).

[43] S. Takeno and M. Gôda, Prog. Theor. Phys. 45, 331 (1971).

[44] L.-J. Hou, Z. L. Mišković, A. Piel, and M. S. Murillo, Phys. Rev. E 79, 046412 (2009).

[45] S. A. Khrapak and B. A. Klumov, Phys. Plasmas 27, 024501 (2020).

[46] R. Nossal, Phys. Rev. 166, 81 (1968).
[47] R. Zwanzig and R. D. Mountain, J. Chem. Phys. 43, 4464 (1965).

[48] P. Schofield, Proc. Phys. Soc. 88, 149 (1966).

[49] S. A. Khrapak, Phys. Plasmas 23, 104506 (2016).

[50] J. A. Anderson, C. D. Lorenz, and A. Travesset, J. Comput. Phys. 227, 5342 (2008).

[51] J. Glaser, T. D. Nguyen, J. A. Anderson, P. Lui, F. Spiga, J. A. Millan, D. C. Morse, and S. C. Glotzer, Comput. Phys. Commun. 192, 97 (2015).

[52] N. P. Kryuchkov, L. A. Mistryukova, V. V. Brazhkin, and S. O. Yurchenko, Sci. Rep. 9, 10483 (2019).

[53] V. V. Brazhkin, Y. D. Fomin, A. G. Lyapin, V. N. Ryzhov, and K. Trachenko, Phys. Rev. E 85, 031203 (2012).

[54] S. A. Khrapak and H. M. Thomas, Phys. Rev. E 91, 033110 (2015).

[55] S. A. Khrapak, Plasma Phys. Controlled Fusion 58, 014022 (2016). 